Portland State University

PDXScholar

Spring 6-2-2016

\title{
Photoemission Electron Microscopy for Analysis of Dielectric Structures and the Goos-Hänchen Shift
}

Theodore Axel Stenmark

Portland State University

Follow this and additional works at: https://pdxscholar.library.pdx.edu/open_access_etds

Part of the Optics Commons

Let us know how access to this document benefits you.

\section{Recommended Citation}

Stenmark, Theodore Axel, "Photoemission Electron Microscopy for Analysis of Dielectric Structures and the Goos-Hänchen Shift" (2016). Dissertations and Theses. Paper 2991.

https://doi.org/10.15760/etd.2992

This Thesis is brought to you for free and open access. It has been accepted for inclusion in Dissertations and Theses by an authorized administrator of PDXScholar. Please contact us if we can make this document more accessible: pdxscholar@pdx.edu. 
Photoemission Electron Microscopy for Analysis of Dielectric Structures and the Goos-

Hänchen Shift

by

Theodore Axel Stenmark

A thesis submitted in partial fulfillment of the requriements for the degree of

Master of Science

in

Physics

Thesis Committee:

Rolf Könenkamp, Chair

Robert Word

Andres LaRosa

Portland State University

216 
(C) 2016 Theodore Axel Stenmark 


\begin{abstract}
Photoemission Electron Microscopy (PEEM) is a versatile tool that relies on the photoelectric effect to produce high-resolution electron images. Ultrafast pulse lasers allow for multi-photon PEEM where multiple visible or IR photons excite a single electron in a nonlinear process. The photoelectron yield in both cases is related to the near-field region of electromagnetic fields at the surface of the sample. We use this ability here to analyze wave propagation in a linear dielectric waveguide with wavelengths of $410 \mathrm{~nm}$ and $780 \mathrm{~nm}$. The propagation constant of the waveguide can be extracted from interference patterns created by light propagating in the waveguide and incident light. Various properties like the polarization dependence of the propagation can be analyzed. The electromagnetic field interaction at the boundaries can then be deduced, which is essential to understand power flow in wave guiding structures. These results match well with simulations using finite element techniques as well as electromagnetic theory.
\end{abstract}




\section{Acknowledgements}

The Author would like to acknowledge Dr. Rolf Könenkamp, Dr. Robert Word and Dr. Joe Fitzgerald for their patients and guidance throughout the project. The images were taken by Dr. Word and he provided significant guidance in analysis and processing of the data. Sample construction in the Focused Ion Beam was performed by Dr. Fitzgerald. This research was supported by the Basic Science Office of the Department of Energy under grant no. DEFG02-07ER46406 


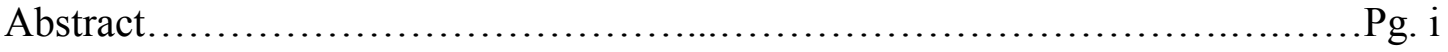

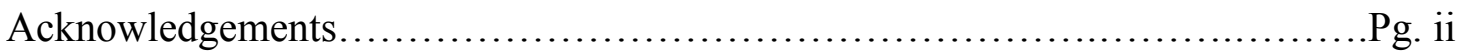

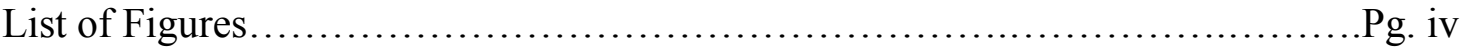

List of Tables................................................................ vi

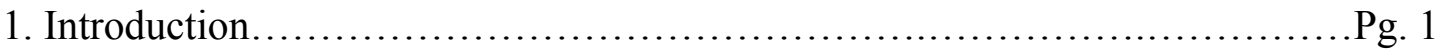

2. Theory of Dielectric Waveguides

2.1 Electromagnetic Theory ............................................ 3

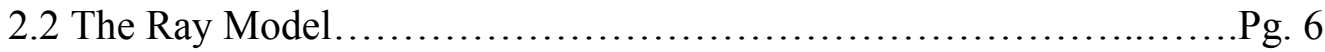

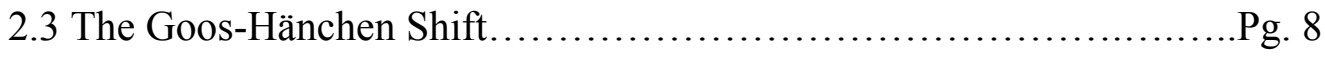

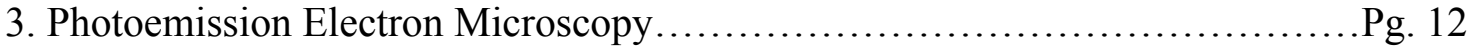

4. Experiment with PEEM

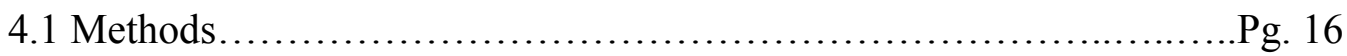

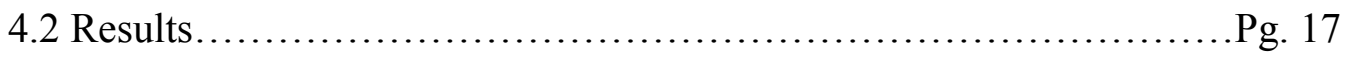

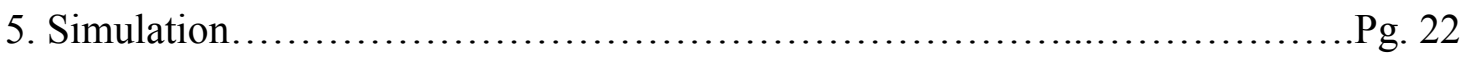

6. Goos-Hänchen Shift Results................................................ 25

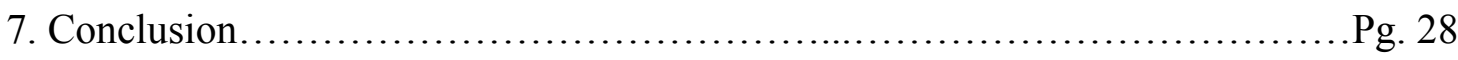

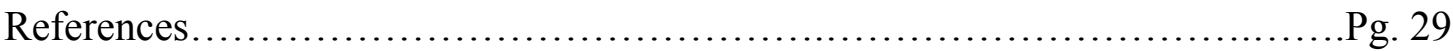




\section{List of Figures}

Figure 1: Basic layout of waveguide geometry (left). The graphical solution for the propagation constant of a $290 \mathrm{~nm}$ thick waveguide with refractive cladding indices of $1,1.5$, and core of 2.14 at a wavelength of $410 \mathrm{~nm}$ (right). The non-asymptotic intersections give the possible guided modes

Figure 2: (a) Ray model without the Goos-Hänchen shift with a round trip fulfilling the transverse resonance condition. (b) Ray model with the Goos-Hänchen shift to account for penetration into the substrate

Figure 3: Simplified Diagram of PEEM. Photoelectrons excited by the laser illumination go through the electron optics in the microscope and hit a phosphor screen producing photons to be recorded by a CCD camera.

Figure 4: Basic waveguide experiment: polarized light is directed towards the milled slit at an incident angle of $60^{\circ}$. Interference between the wave-guided light and the non-scattered light produces an interference pattern

Figure 5: PEEM images of the waveguide illuminated by TE (top) and TM (bottom) polarized light with wave lengths of $410 \mathrm{~nm}$ (left) and $780 \mathrm{~nm}$ (right).

Figure 6: Periodograms from the waveguide under 410-nm illumination. Top is TE and bottom is TM polarization. Left is the milled and right is the bulk region. FFT powers normalized to one for largest peak. (1,2) Main propagating modes. (3) Interference of main modes. (4) higher order multiphoton emission peaks

Figure 7: Periodograms of the $\lambda=780 \mathrm{~nm}$ illuminated waveguide. The only significant peak is the single propagating mode.

Figure 8: By shifting the data sampling location we observe the disappearance of the strongly suppressed "third" mode. 
Figure 9: The mode excited by the first wave front is interfered with by the second wave front at

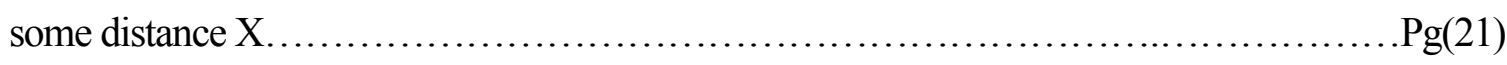

Figure 10: (a) Time averaged electric field in finite element technique simulation using COMSOL multiphysics. (b) Close up of the guiding region supporting two modes. (c) Line graph of the electromagnetic field from the surface of the ITO layer.

Fig. 11: Simulated (red) vs. experimental (blue) data for TE and TM polarizations in the bulk and milled regions at $410 \mathrm{~nm}$ excitation.

Figure 12: (a) Semi-log line graph of electromagnetic field intensity taken across (b) a COMSOL simulated waveguide with $\mathrm{h}=240 \mathrm{~nm}, \lambda=780 \mathrm{~nm}, \mathrm{n}_{1}=1.78, \mathrm{n}_{2}=1.53, \mathrm{n}_{3}=1$. The graph is used to determine the penetration depth at each interface.

Figure 13: Goos-Hänchen shift vs. angle of propagation at $410 \mathrm{~nm}$ for both interfaces and polarizations in the milled region. Solid line (blue) is the theoretical shift at the air interface. Dashed line (red) is the theoretical shift at the glass interface. Squares represent experimentally determined data points at air interface (black) and glass interface (purple). Similarly circles are data points from simulation 


\section{List of Tables}

Table 1: Measurements for TE $\lambda=410 \mathrm{~nm}$ modes in milled and bulk regions. ............Pg(25)

Table 2: Measurements for TM $\lambda=410 \mathrm{~nm}$ modes in milled and bulk regions. ..............Pg(25)

Table 3: Measurements for $\lambda=780 \mathrm{~nm}$ for TE and TM modes in milled regions. ........... $\operatorname{Pg}(26)$ 


\section{Introduction}

Much of the world's communication technology relies on optical devices. Fiber optic cables provide fast and reliable telecommunication, dielectric resonators serve as antennas, and optical disc drives store our data. Among optical components the dielectric waveguide is of particular importance for communications and computing and is needed for integrated optical circuits [1]. The waveguide consists of a simple slab like structure that employs total internal reflection to confine light in a dielectric layer surrounded by materials of lower refractive indexes. When the dielectric layer is thin, on the order of hundreds of nanometers, it only allows for a few distinct resonances to propagate in the waveguide. With the knowledge of these modes a complete picture of the energy propagation and optical properties of the dielectric structure can be developed.

As light propagates in a waveguide a polarization dependent shift occurs upon reflection at the interface of the dielectric waveguide and surrounding materials. This shift happens because in a dielectric waveguide the light is not fully contained in the core region, but rather the electric field bleeds into the surrounding materials in an evanescently decaying wave. This phenomenon is known as the Goos-Hänchen shift [2], which is a lateral shift of a finite beam due to the dependence of the evanescent field on the incident angle of the light. The magnitude of the Goos-Hänchen shift affects the allowed modes of propagation.

The Goos-Hänchen shift is important for understanding the nature of light interactions at material boundaries and can be incorporated into the ray model of light for improved accuracy [3]. The shift is generally on the order of a wavelength in materials with real dielectric constants but through the use of meta-materials much larger shifts can be induced $[4,5]$. These shifts have potential applications in optical sensors [6] and optical switching devices [7]. 
Furthermore, negative shifts can be obtained using materials with large imaginary refractive indexes such as metals [8] or by employing meta-materials like photonic crystals [9]. The ability to control light in this fashion could lead to new photonic devices for communication and computing.

Photoemission Electron Microscopy (PEEM) is a powerful surface imaging technique for observing electromagnetic near-field phenomena on the nanoscale. By directing high intensity light onto a sample, incident photons excite electron emission via the photoelectric effect. The photoelectrons are used to produce high quality images, which have obtained resolution down to $5 \mathrm{~nm}$ in biological samples [10]. The contrast of the image comes directly from the spatial electron yield emitted from the samples surface, which depends on the work function, surface electron density, and topography of the material as well as the energy and intensity of the incident photons.

We demonstrate that PEEM can experimentally determine the propagating modes in a simple dielectric waveguide consisting of materials with real indexes of refraction to a high degree of accuracy. Because of the discrete modes of propagation, the incident excitation beam creates a standing interference pattern with the confined light. The interference creates strong variation in electromagnetic fields, which multi-photon PEEM is ideally suited to detect. The high-resolution surface information of PEEM not only allows for analysis of the wave propagation but also provides insight to fundamental aspects of light propagation in dielectrics when combined with electromagnetic theory. Understanding the propagation and energy confinement in these nanostructures is imperative to the development of the next generation of photonic devices. 


\section{Theory of Dielectric Waveguides}

\subsection{Electromagnetic Theory}

The propagation of light in a dielectric slab waveguide can be understood through electromagnetic theory. For the dielectric waveguide case we may use the source free time dependent version of the equations:

$$
\begin{aligned}
& \nabla \cdot D=0 \\
& \nabla \cdot B=0 \\
& \nabla \times E=-\frac{\partial B}{\partial t} \\
& \nabla \times H=-\frac{\partial D}{\partial t} .
\end{aligned}
$$

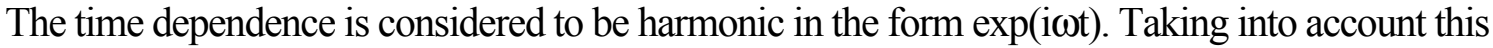
time dependence Eqs. $(3,4)$ become:

$$
\begin{aligned}
& \nabla \times E=-i \omega B, \\
& \nabla \times H=i \omega D .
\end{aligned}
$$

The geometry of our structure as seen in Fig. 1 will be considered as follows, an infinite dielectric slab in the $y, z$ plane bounded at $x$ equal to 0 and $x$ equal to $h$ by two different dielectrics. The propagation of modes in the slab will be considered to be in the $\mathrm{z}$ direction.

So long as there is no surface charge, the tangential components of $\mathbf{E}$ and $\mathbf{H}$ parallel to the interface of the waveguide must be continuous across the core-cladding boundary. These boundary conditions can be taken advantage of to find the modes allowed to propagate inside the dielectric structure. Since any field can be decomposed into components with transverse electric (TE) and transverse magnetic (TM) polarization, we can split the problem in two and solve for each polarization separately. For TE polarization the $H_{y}, E_{x}$, and $E_{z}$ components of the electromagnetic field are 0 . This is highly convenient as the cross product of $\mathbf{E}$ in Eq. (5) may be simplified to

$$
\frac{d E_{X}}{d y}-\frac{d E_{y}}{d x}=i \omega \mu H_{z}
$$


Now we will take advantage of our geometry. Since the structure is uniform along the y-axis, the partial derivative with respect to the $y$-coordinate is zero. This leaves the $z-$ component of $\mathbf{H}$ proportional to the first derivative of the E-field. Recall that the boundary conditions are that the component of $\mathbf{E}$ and $\mathbf{H}$ parallel to the interface must be continuous. Since the transverse component of $\mathbf{E}$ is only $E_{y}$ and the parallel component of $\mathbf{H}$ is proportional to the first derivative of $E_{y}$ the boundary conditions may be put solely in terms of $E_{y}$ and its derivative with respect to $\mathrm{x}$.

Next we introduce the homogeneous wave equation,

$$
\nabla^{2} E+k_{0}^{2} n^{2} E=0
$$

where $n$ is the refractive index of the medium, $k_{0}$ is the free space wave number and it is assumed that any time dependence is harmonic. The spatial modulation of a particular mode in the direction of propagation, the $\mathrm{z}$ direction, is considered to be $\exp (\mathrm{i} \beta \mathrm{z})$, where $\beta$ is the propagation constant of that mode, which when divided by the free space wave vector, gives the effective index of the guided wave, $N_{\text {eff }} \equiv \beta / k_{0}$. Also noting again that the derivative with respect to $\mathrm{y}$ is 0 from the geometry, our wave equation, becomes,

$$
\frac{\partial^{2} E}{\partial x^{2}}+\left(k_{0}^{2} n_{i}^{2}-\beta^{2}\right) E=0
$$

The index on $n_{i}$ indicates the dielectric layer in the structure. The wave equation in each layer is then given as:

$$
\begin{array}{lll}
\frac{\partial^{2} E_{y}}{\partial x^{2}}=s^{2} E_{y} ; & x \leq 0 ; & s^{2}=\beta^{2}-k_{0}^{2} n_{3}^{2}, \\
\frac{\partial^{2} E_{y}}{\partial x^{2}}=q^{2} E_{y} ; & 0 \leq x \leq h ; & \mathrm{q}^{2}=k_{0}^{2} n_{1}^{2}-\beta^{2}, \\
\frac{\partial^{2} E_{y}}{\partial x^{2}}=p^{2} E_{y} ; & \mathrm{h} \leq x ; & \mathrm{p}^{2}=\beta^{2}-k_{0}^{2} n_{2}^{2} .
\end{array}
$$


To have a guided mode the electromagnetic field must be contained within the core of the waveguide. With this in consideration we take solutions of exponential decaying evanescent fields in the cladding and an oscillatory solution in the core in the form:

$$
\begin{aligned}
& E_{y}=D \exp (s x) ; \quad \mathrm{x} \leq 0, \\
& E_{y}=D \cdot \cos (q x)+F \cdot \sin (q x) ; \quad 0 \leq x \leq h, \\
& E_{y}=[D \cdot \cos (q h)+F \cdot \sin (q h)] \exp (-p(x-h)) ; \quad h \leq x .
\end{aligned}
$$

These fields must fulfill the boundary conditions obtained previously for the transverse component of the electric field and its first derivative. Taking the derivative with respect to $\mathrm{x}$ for each section yields:

$$
\begin{aligned}
& \frac{\partial E_{y}}{\partial x}=s D \exp (s x) ; \quad x \leq 0, \\
& \frac{\partial E_{y}}{\partial x}=q(-D \cdot \cos (q x)+F \cdot \sin (q x)) ; \quad 0 \leq x \leq h, \\
& \frac{\partial E_{y}}{\partial x}=-p[D \cdot \cos (q h)+F \cdot \sin (q h)] \exp (-p(x-h)) ; \quad h \leq x .
\end{aligned}
$$

The coefficients $D$ and $F$ can be related by matching conditions at the $\mathrm{x}=0$ boundary,

$$
\begin{aligned}
& s D \exp (s x)=q(-D \cdot \cos (q x)+F \cdot \sin (q x)), \\
& s D=q F .
\end{aligned}
$$

Matching at the conditions at $\mathrm{x}=\mathrm{h}$ we obtain:

$$
\begin{aligned}
& q(-D \cdot \cos (q x)+F \cdot \sin (q x))=-p[D \cdot \cos (q h)+F \cdot \sin (q h)] \exp (-p(x-h)) \\
& q(-D \cdot \cos (q x)+F \cdot \sin (q x))=-p(D \cdot \cos (q h)+F \cdot \sin (q h)) .
\end{aligned}
$$

We can reduce the system to an eigenvalue equation in terms of $p, q$ and $s$ utilizing Eqs. (13-14).

$$
\begin{aligned}
& q\left(-\frac{q}{s} F \cdot \sin (q h)+F \cdot \cos (q h)\right)=-p\left(\frac{q}{s} F \cdot \cos (q h)+F \cdot \sin (q h)\right), \\
& q\left(-\frac{q}{s} \cdot \tan (q h)+1\right)=-p\left(\frac{q}{s}+\tan (q h)\right) \\
& q+\frac{p q}{s}=\tan (q h)\left(\frac{q^{2}}{s}-p\right) \\
& \tan (q h)=\frac{q(s+p)}{q^{2}-p s}
\end{aligned}
$$


Recall that $p, q$, and $s$ as defined in Eq. (10) are all dependent on only propagation constant, refractive index of dielectric layer, and free space wave number. This equation can then be graphically solved as in Fig. 1. for the propagation constant with the known refractive indices of the materials and excitation wavelength.
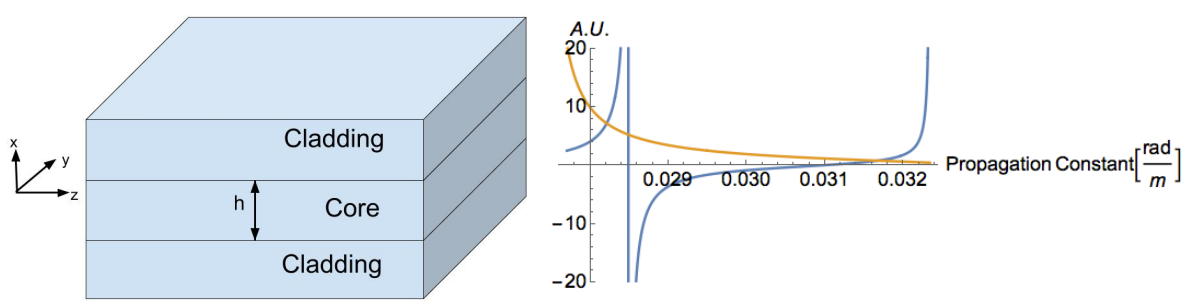

Figure 1: Basic lay out of waveguide geometry (left). The graphical solution for the propagation constant of a $290 \mathrm{~nm}$ thick waveguide with refractive cladding indices of 1 , 1.5 , and core of 2.14 at a wavelength of $410 \mathrm{~nm}$ (right). The non-asymptotic intersections give the propagation constants for the possible guided modes.

For the TM modes $E_{y}, H_{x}$, and $H_{z}$ are zero and the boundary conditions are put only in terms of $H_{y}$ through Eq. (6) and the derivation proceeds in a similar fashion. A complete derivation may be found in reference [11].

\subsection{The Ray Model}

An alternate approach to calculating the propagation constant of a dielectric waveguide is through a ray model using the transverse resonance condition. This method uses ray optics to find the discrete allowed modes of propagation assuming total internal reflection at each interface causing the ray to bounce back and forth in a zigzag fashion as in Fig. 2. This ray can be broken down into its transverse and longitudinal components. The transverse resonance condition states that a mode can only propagate in the wave guide if the transverse component interferes constructively with its self, that is, it undergoes a phase that is a multiple of $\pi$ on a trip from one side of the guide to the other. 
On this path one must consider the thickness of the guiding structure, $h$, the angle at which the wave is zigzagging, $\theta$, and the phase shift, $\phi$, that is obtained upon total internal reflection at the interface. Together these give us our condition,

$$
h k_{0} n_{1} \cdot \cos (\theta)-\phi_{1}-\phi_{2}=m \pi
$$

where $\mathrm{k}_{0}$ is the free space wavenumber, $n_{l}$ is the refractive index of the material, and $\mathrm{m}$ is some positive integer. The phase shifts can be obtained from the well-known Fresnel equations. They are polarization dependent and given by:

$$
\begin{gathered}
\phi_{T E}=\tan ^{-1}\left[\frac{\left(n_{\text {core }}^{2} \cdot \sin ^{2} \theta-n_{\text {cladding }}^{2}\right)^{1 / 2}}{n_{\text {core }} \cdot \cos \theta}\right] ; \\
\phi_{T M}=\tan ^{-1}\left[\frac{\left(n_{\text {core }}^{2} \cdot \sin ^{2} \theta-n_{\text {cladding }}^{2}\right)^{1 / 2}}{n_{\text {core }} \cdot \cos \theta} \cdot \frac{n_{\text {core }}^{2}}{n_{\text {cladding }}^{2}}\right] .
\end{gathered}
$$

Where $n_{\text {core }}$ is the refractive index of the core material of the waveguide and $n_{\text {cladding }}$ is the index of the outer material as seen in Fig. 2. Once again we can produce an eigenvalue equation that depends on refractive index, free space wavelength, waveguide thickness, and angle of propagation. Given the other parameter we can determine the angle and thus the propagation constant.

The ray model can hold several advantages for pictorial understanding as well as computational simplicity. The simple ray model, however, is not a good representation for structures with a scale on the order of the wavelength. While it can provide accurate results for the propagation constant it neglects important features such as the evanescent field. Since we know the electric field extends into the cladding region of the waveguide it would seem more reasonable to including this light penetration in a ray model as in Fig. 2(b) as first pointed out by Burke in 1971 [12]. In this refined ray model the waveguide thickness is effectively increased by 
the depth the ray penetrates which corresponds to the characteristic decay length of the evanescent wave in the cladding [13].
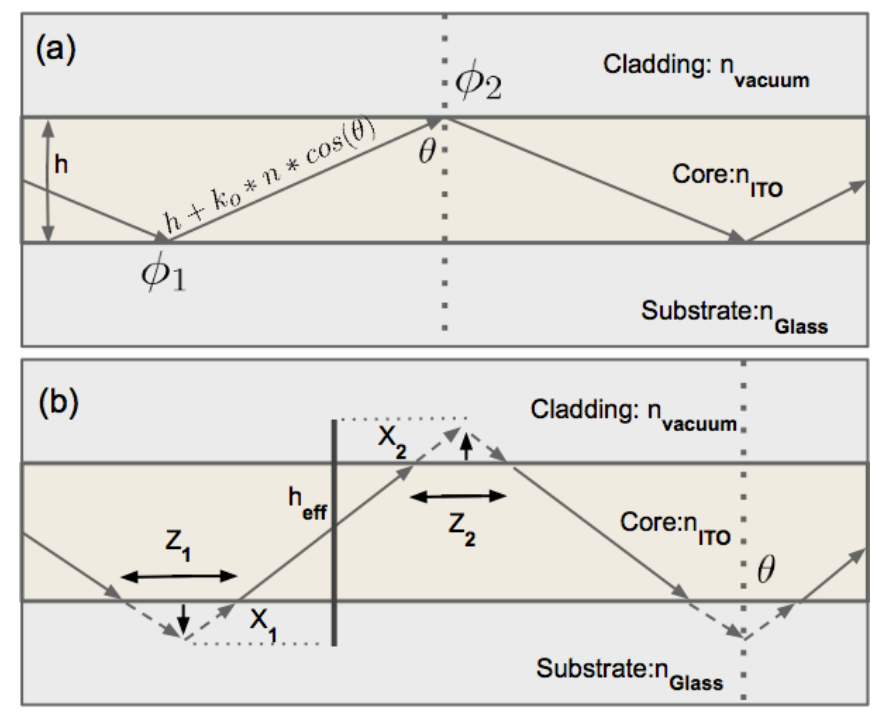

Figure 2. (a) Ray model without the Goos-Hänchen shift with a round trip fulfilling the transverse resonance condition. (b) Ray model withthe Goos-Hänchen shift to account for penetration into the substrate.

This model has been shown to match with the electromagnetic theory [14] while keeping propagation along the lateral direction constant due to the time delay between the entering and exiting of the ray. The refined ray picture can be used as a reasonable model to understand the energy flow and power transfer in coupled wave guiding structures $[15,16]$. As seen in Fig. 2(b) the ray now undergoes an apparent lateral shift upon reflection. This is a phenomenon that was first predicted by Newton [17] and has come to be known as the GoosHänchen shift, after it was experimentally demonstrated by Goos and Hänchen in 1947 [2].

\subsection{The Goos-Hänchen Shift}

The Goos-Hänchen shift is the lateral shift of a finite light ray in the plane of reflection. The root of this phenomenon is the fact that reflection at an interface has an angular dependence. This can be seen in phase shifts in Eq. $(23,24)$. Because a finite beam is composed 
of a superposition of plane waves, each with a different angular component, the components reflect differently effectively shifting the center of the beam.

A formula for the magnitude of the shift, as first obtained by Artmann in 1948 [18], can be obtained by considering the decomposition into plane wave components. To begin consider two plane waves incident at slightly different angles. The complex amplitude at the interface of reflection may be written as,

$$
A(z)=[\exp (i \Delta \beta z)-\exp (-i \Delta \beta z)] \exp (-i \beta z)
$$

where $\beta$ is the propagation constant of the wave. The phase shift that occurs at the interface is a function of the propagation constant. Assuming that the difference in phase shift and propagation constant of our two plane waves is small we may expand the phase shift as,

$$
\Phi(\beta+\Delta \beta)=\Phi(\beta)+\frac{d \Phi}{d \beta} \Delta \beta
$$

Then the reflected ray packet may be written as:

$$
\begin{gathered}
B(z)=\left[\exp \left(i \Delta \beta z-\frac{\partial \Phi}{\partial \beta} \Delta \beta\right)+\exp \left(-i \Delta \beta z+\frac{\partial \Phi}{\partial \beta} \Delta \beta\right)\right] \exp (-i(\beta z-\Phi)) \\
B(z)=\cos \left(\Delta \beta\left(z-\frac{\partial \Phi}{\partial \beta}\right)\right) \exp (-i(z \beta-\Phi))
\end{gathered}
$$

Then the displacement of the reflected beam, $z$, is simply

$$
z=\frac{\partial \Phi}{\partial \beta}
$$

where $\Phi$ is the Fresnel phase shift. This gives us a polarization-dependent equation for the lateral displacement of the beam.

$$
z_{T E}=2\left(n_{\text {core }}^{2} \cdot \sin ^{2} \theta-n_{\text {cladding }}^{2}\right)^{-1 / 2} \tan \theta \frac{1}{k_{0}}
$$




$$
z_{T M}=z_{T E}\left(\frac{n_{\text {core }}^{2} \cdot \sin ^{2} \theta}{n_{\text {cladding }}^{2}}+\sin ^{2} \theta-1\right)
$$

These results give a good value for the shift but break down as the beam comes within half a degree of the critical angle due to our approximation in Eq. (25). Other formulations have been proposed using approaches such as conservation of energy [19,20,21], but Artmann's formulation remains the simplest and provides an extremely good approximation for incidence far from the critical angle. For the applications of the Goos-Hänchen shift explored here, the Artmann approach is sufficient.

To show that extended ray model is consistent we must demonstrate an equivalent shift. In the extended version we claim the ray penetrates some distance, $\mathrm{x}$ into the substrate, which through simple geometry gives a shift of,

$$
z=2 x \cdot \tan \theta,
$$

The penetration depth, $\mathrm{x}$, is closely related to the transverse decay constant.

Because the field in the cladding is evanescent, it decays exponentially away from the core of the waveguide. This decay is described by,

$$
\gamma=k_{0}\left(n_{\text {core }}^{2} \cdot \sin ^{2} \theta-n_{\text {cladding }}^{2}\right)
$$

The penetration depth of the ray is then given by:

$$
x=\frac{1}{\gamma q}
$$

where $q$ is a polarization dependent factor that can be derived based on the corresponding boundary conditions and written as,

$$
\begin{gathered}
q_{T E}=1 ; \\
q_{T M}=\frac{n_{\text {core }}^{2} \cdot \sin ^{2} \theta}{n_{\text {cladding }}^{2}}+\sin ^{2} \theta-1 .
\end{gathered}
$$


Comparing the results given by Eqs. (32-36), we see it is precisely identical to the equations obtained in the plane wave expansion method. 


\section{Photoemission Electron Microscopy}

The original concept of using photoelectrons to produce images is credited to Brüche who, in 1933, demonstrated that a primitive magnetic lens could be used to produce an image from accelerated photoelectrons [22]. Though a number of interesting studies were conducted with early versions of PEEM, it was not until the invention of a reliable ultrahigh vacuum system (UHV) that it became a competitive technique [23]. Before UHV, vacuum systems mainly used oil diffusion pumps, which introduced a large amount of contamination into the system. This is especially detrimental to PEEM as photoelectrons can only escape from the very surface of the sample. One of the early UHV-PEEM instruments was constructed by the Oregon microscope project at the University of Oregon [24]. The construction of this instrument was spearheaded by Griffith and Rempfer and was primarily used for biological applications achieving spatial resolution down to $10 \mathrm{~nm}[25,26,27]$. Gertrude Rempfer was one of the pioneers in the development of PEEM and her theoretical work drove development for many years [28].

PEEM can employ either incoherent ultraviolet (UV) radiation sources such as mercury lamps or X-rays generated by a synchrotron. The high photon energies of these sources generally allow for a single photon to produce electron emission from the photoelectric effect. This type of PEEM is referred to as single photon PEEM, or 1P-PEEM. As one would expect, UV lasers can dramatically increase the photoelectron yields by increasing the intensity of illumination. The work function of most materials prohibits 1P-PEEM from studying interesting optical phenomena that occur in the visible and infrared spectral region. With the high intensities of ultrafast pulse lasers, it is possible to produce sufficient photoemission using two (2P-PEEM) or even three (3P-PEEM) low energy photons to overcome the work function. 
Because multiphoton emission is a nonlinear response the process is particularly sensitive to changes in photon density. This method has proved useful in the study of lightconfining mechanisms such as surface plasmon polaritons [29,30], which are collective oscillations of electrons and photons on metal surfaces. Plasmonic responses have attracted considerable attention as plasmonics provides a way to confine light past the diffraction limit. PEEM has extensive potential for this application as it provides direct imaging of the optical nearfield and is a non-invasive imaging technique. In addition to studying surface plasmon polaritions, the multiphoton process is also well suited for observing electromagnetic fields on the surface of photonic structures consisting of dielectric materials with real dielectric constants [31]. In the past years we have used multiphoton excitation to characterize optical wave propagation in nanostructured optical waveguides [32].

The general basic design of the modern PEEM is remarkably similar to Brüche's original instrument but with more refined components. One of the major improvements to the modern instrument is correction of the aberration. Due to the energy spread and spatial distribution of the photoelectrons emitted from the sample both spherical and chromatic aberrations are introduced by the accelerating field at the cathode and electron lenses. An electron mirror can introduce aberrations approximately inverse to these thereby reducing the total aberration to first order [33]. One of Rempfer's major contributions was her testing and development of an electron mirror to correct for spherical and chromatic aberrations [34]. The electron mirror is held at a large potential difference to reflect the electron beam with adjustable electrodes to control the electric field and adjust the aberration introduced by the mirror. Aberrations can theoretically be reduced even further by going from a diode mirror to a three or four electrode mirror giving more freedom to adjusting the parameters $[35,36]$. 
An outline of the basic design of the electron optics in our aberration-corrected microscope is seen in Fig. 3. A laser beam illuminates the sample at an incident angle of $60^{\circ}$ and excites the photoelectrons emitted at low energy. These electrons are accelerated through a voltage difference of approximately $20 \mathrm{keV}$ to the objective lens. The objective lens and auxiliary lens form a zoom pair, which controls the initial magnification. The objective lens is largely responsible for the final resolution of the final image, as aberration introduced by the objective lens will be magnified in following transfer lenses.

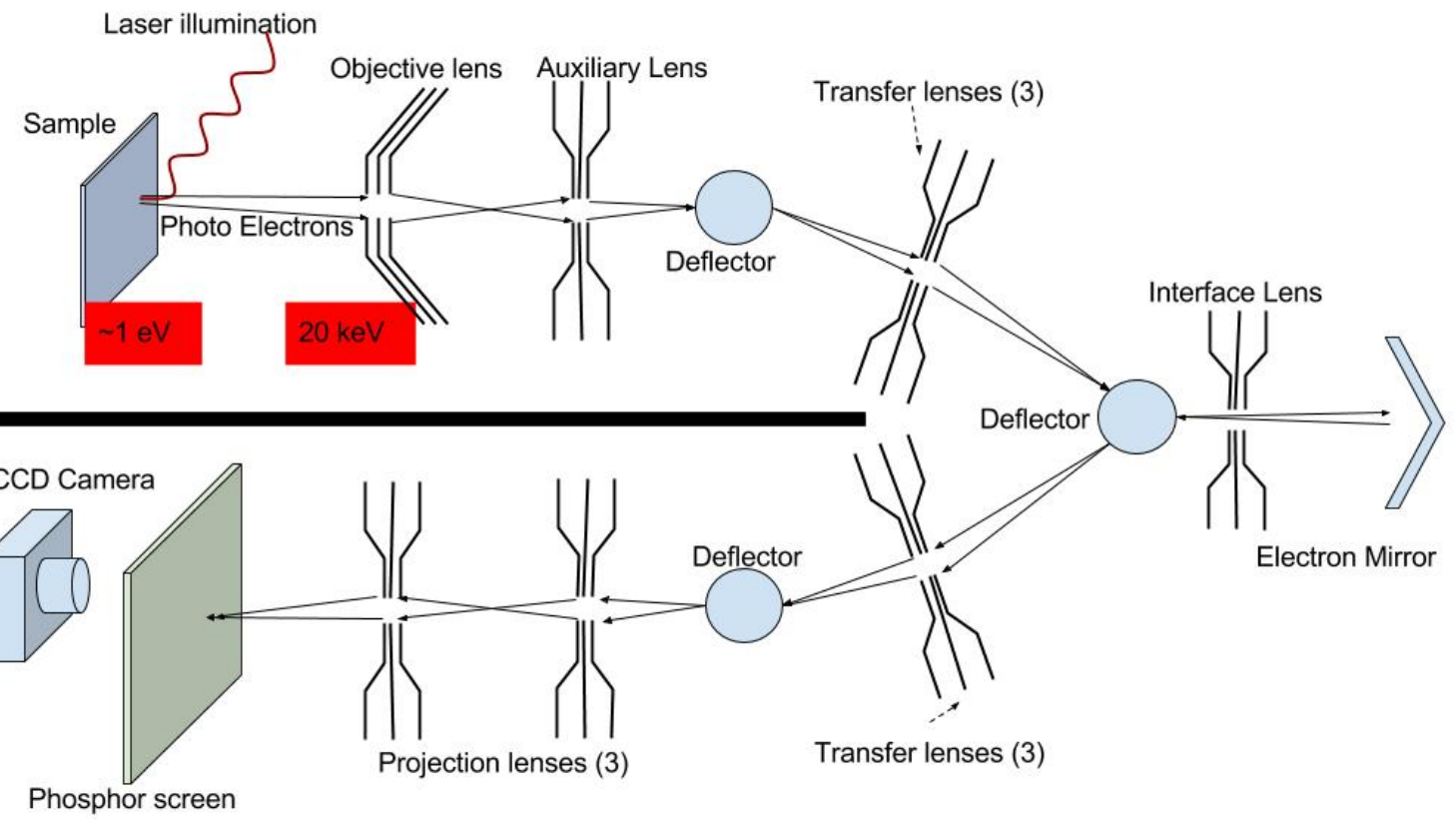

Figure 3. Simplified diagram of a PEEM. Photoelectrons excited by the laser illumination go through the electron optics in the microscope and hit a phosphor screen producing photons to be recorded by a CCD camera.

An aperture is located on the optical axis in the back focal plane of the objective lens to limit the angular spread of electrons. The aperture angle is directly related to the maximum obtainable resolution based on the diffraction limit [37]. The $30 \mu \mathrm{m}$ aperture in our system has an aperture angle of approximately $0.4^{\circ}$, which gives a theoretical maximum achievable resolution 
on the order of a few nanometers [36]. Currently PEEM is far from this resolution, with aberration being the limiting factor rather than the diffraction limit.

After the objective lens and aperture, the transfer lenses and magnetic deflectors are used to guide the electron beam through the optical column to the electron mirror. The potential of the electron mirror is chosen such that the reflected beam is coincident with the incident beam, but reverse in direction, i.e. the electron beam is reflected back through the magnetic deflector, which then deflects it such that a separation between incident and reflected beam occurs. The beam is guided back through a second set of transfer lenses to the projection lens, which magnifies and projects the final image onto a phosphor screen. When each electron strikes the phosphor it emits many photons, providing a kind of signal amplification due to the high electron energy. These photons are imaged in an optical transfer lens system to a CCD camera to record the final image. 


\section{Experiment with PEEM}

\subsection{Methods}

The sample consisted of a $0.2 \mathrm{~mm}$ borosilicate glass sheet coated with an indiumtin-oxide (ITO) layer of $\sim 290 \pm 20 \mathrm{~nm}$ thickness acquired from SPI supplies, Inc. The refractive index of ITO is $2.14 \pm 0.01$ at a wavelength $\lambda=410 \mathrm{~nm}$ and $\mathrm{n}=1.78 \pm 001$ at $\lambda=780 \mathrm{~nm}$ [38].

Other optical properties of ITO thin films such as absorption are dependent on the thickness of the film [39]. The real part of the refractive index of the glass substrate is $1.53 \pm .01$, the imaginary index is negligible and may be disregarded with no significant consequence. Parallel strip waveguides were defined with a FEI Strata 273 focused ion beam (FIB). A deep narrow groove was milled to a depth of $\sim 240 \mathrm{~nm}$ into the sample with dimensions of $6.0 \mu \mathrm{m} \times 0.4 \mu \mathrm{m}$ to allow for in-coupling of light into the waveguide through diffraction. A thinned waveguide region was milled to a depth of $\sim 50 \mathrm{~nm} \pm 10 \mathrm{~nm}$ and dimensions of $2.0 \mu \mathrm{m} \times 5.0 \mu \mathrm{m}$ connecting to the center portion of the groove.

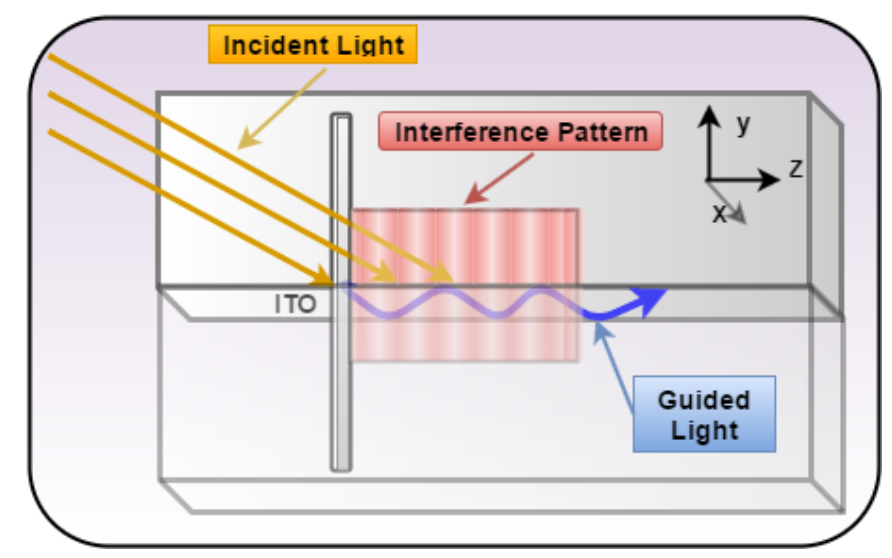

Figure 4. Basic waveguide experiment: polarized light is directed towards the milled slit at an incident angle of $60^{\circ}$. Interference between the wave-guided light and the nonscattered light produces an interference pattern.

As shown in Fig. 4, the sample was oriented in the PEEM such that the groove was perpendicular to the incident laser light. The optical excitation beam was incident at an angle 
of $60^{\circ}$ from the sample normal. The waveguide illuminated at wavelengths of $\lambda=410 \mathrm{~nm}$ and $\lambda=$ $780 \mathrm{~nm}$. In this configuration light is coupled into the groove of the waveguide and propagates laterally in through the waveguide. This guided light interferes with the direct excitation light and generates a stationary interference pattern. This pattern is imaged in the PEEM with a spatial resolution of $\sim 30 \mathrm{~nm}$ and can be analyzed in terms of the propagation constants of the waveguided light.

The Spectra-Physics Mai Tai Ti:Sapphire laser used in the experiment generates 80-fs pulses with energies in the low $\mathrm{nJ}$ range at a rate of $80 \mathrm{MHz}$. A Del Mar second harmonic generator was used to up-convert the IR pulses to $410 \mathrm{~nm}$. The $\mathrm{CW}$-equivalent power at $780 \mathrm{~nm}$ was $900 \mathrm{~mW}$ and at $410 \mathrm{~nm}$ it was $50 \mathrm{~mW}$.

With a work function of $4.2 \mathrm{eV}$ [40], ITO requires a 2-photon process at $\lambda=410$ $\mathrm{nm}(3.02 \mathrm{eV})$ to produce an electron emission. At $\lambda=780 \mathrm{~nm}$ the photon energy drops to $1.59 \mathrm{eV}$ requiring a three photon excitation process. For imaging at $780 \mathrm{~nm}$ the sample only exhibited interference patterns in the milled region while the smooth un-milled region had no visible photoemission. The increased emission in the milled area is attributed to enhancement of the electric field due to surface roughness. The milled region at $780 \mathrm{~nm}$ illumination only produced images for a short time period before the contrast disappeared. We suspect this to be due to surface smoothing and annealing in the intense illumination. Gallium residue from the FIB process may play a role as well, however its contribution is not well understood.

\subsection{Results}

The two areas of interest are the central area referred to as the milled region and the section on either side referred to as the bulk region as seen in Fig. 5. The brightness in the 
image is proportional to the photoelectron yield, which relates to the electric field in the sample

as,

$$
Y P E \propto\left\|E_{t o t}\right\|^{2 n},
$$

where $n$ is the order of the photoemission process i.e. $n=2$ for $\lambda=410 \mathrm{~nm}$ light and $n=3$ for $\lambda=$ $780 \mathrm{~nm}[32]$.
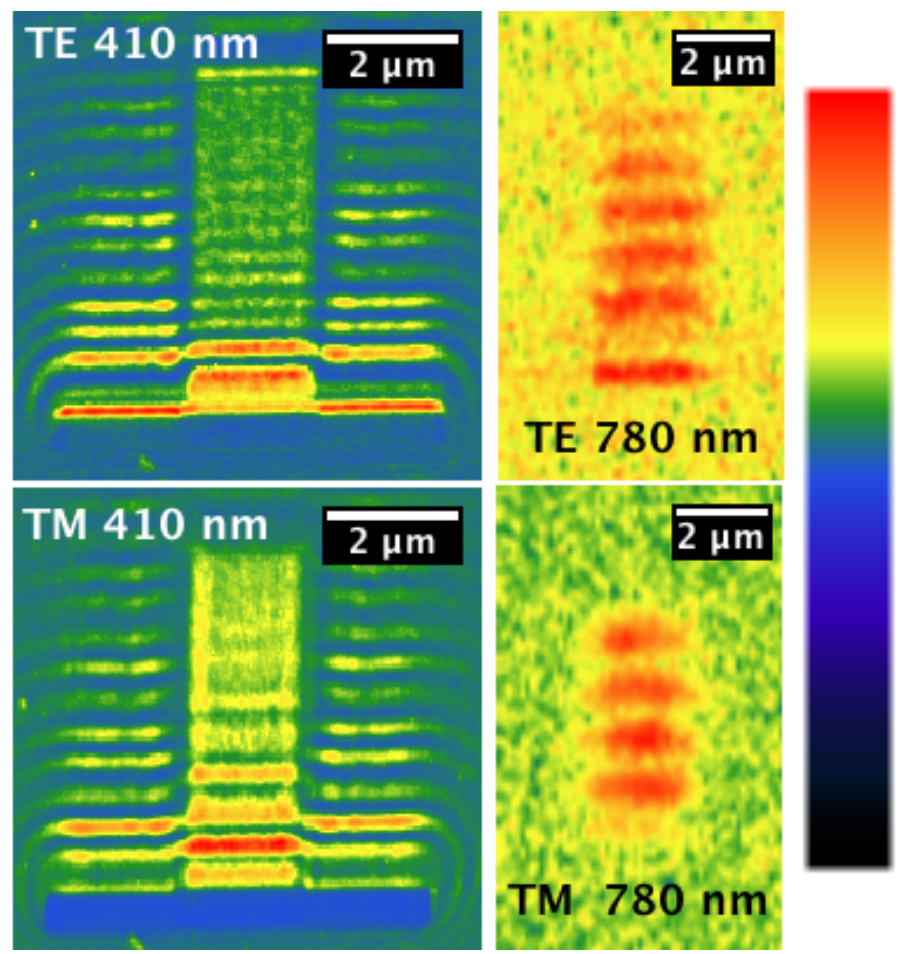

Figure 5. PEEM images of the waveguide illuminated by TE (top) and TM (bottom) polarized light with wavelengths of $410 \mathrm{~nm}$ (left) and $780 \mathrm{~nm}$ (right).

To obtain the periodic information of the interference patterns, we average the pixel value perpendicular to the propagation path and take the Fourier transform of the resulting spatial intensity profile after applying a Hamming window to the data. The Fourier transform provides the spatial frequency spectrum of the interference pattern. Dividing the length of the waveguide by the frequency converts it to periodic data as seen in Figs. 6 and 7 .

For the $410 \mathrm{~nm}$ illumination, theory predicts two propagating modes, while the $780 \mathrm{~nm}$ illumination supports only one mode. The two main peaks in the $410 \mathrm{~nm}$ periodograms 
represent these main guided modes allowed by the parameters of the waveguide labeled 1 and 2 . In addition to the main modes of propagation several other features appear in the 410-nm periodograms. The interference between the two propagating modes creates a beating pattern in the interference seen between 1 and $2 \mu \mathrm{m}$ in the bulk region and labeled 3 . This mode is lost in the TE milled region in the windowing threshold.

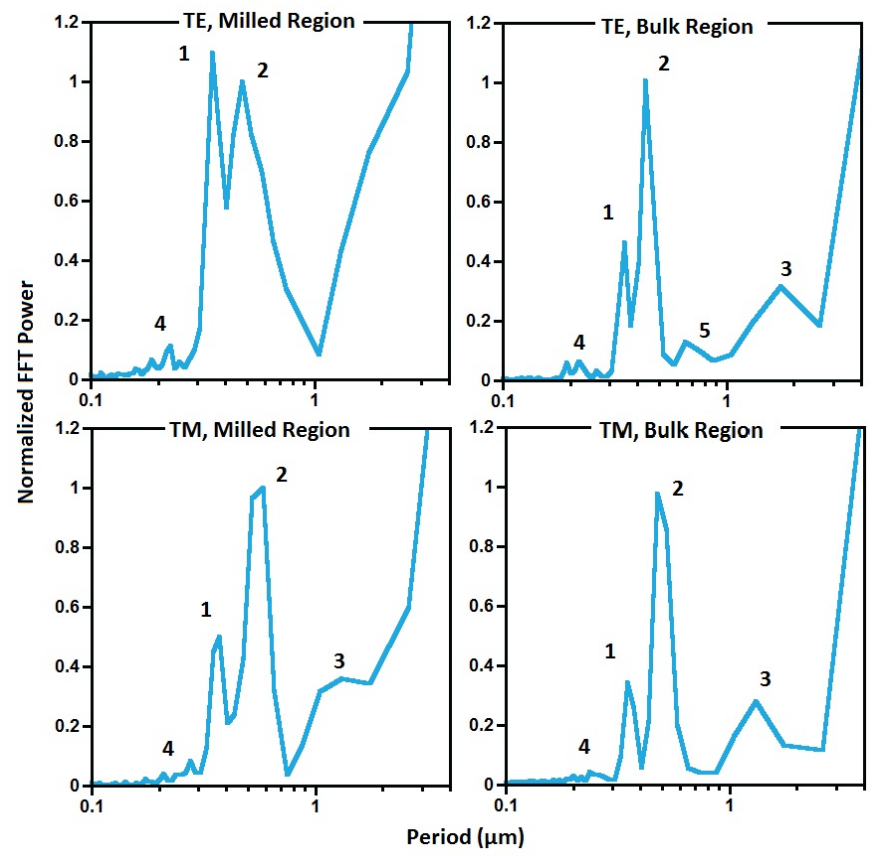

Figure 6. Periodograms from the waveguide under 410-nm illumination. Top is TE and bottom is TM polarization. Left is the milled and right is the bulk region. FFT powers normalized to one for largest peak. $(1,2)$ Main propagating modes. (3) Interference of main modes. (4) higher order multi-photon emission peaks. (5) strongly suppressed $3^{\text {rd }}$ mode.
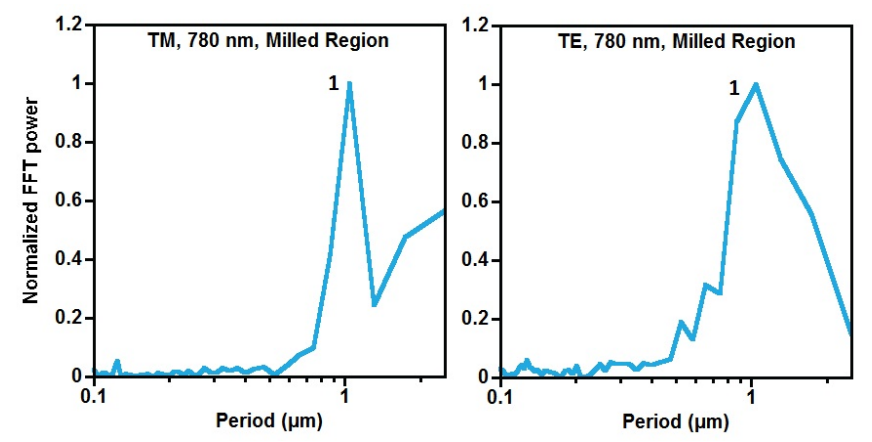

Figure 7. Periodograms of the $\lambda=780 \mathrm{~nm}$ illuminated waveguide. The only significant peak is the single propagating mode. 
Small peaks visible just above the noise level, labeled 4 are present as well. These small peaks are the result of the non-linearity of the multi-photon emission process [32]. The total electromagnetic field is the interference of the propagation modes and incident light given by,

$$
E_{\text {total }}=E_{\text {incident }}+E_{\text {mode } 1}+E_{\text {mode2 }}
$$

The photoemission yield in nP-PEEM is proportional to the $n$th power of the sum of the different fields as in Eq. (37). Putting Eq. (38) in Eq. (37) produces cross terms for two- and three- photon emission. Because the total photoemission is directly related to the electromagnetic field, each term corresponds to a peak in the periodogram. The higher order terms are weaker and correspond to the peaks labeled 4, which appear in the Fourier analysis.

The last unexplained feature, labeled 5, appears only in the TE bulk region at a periodicity of $\sim 700 \mathrm{~nm}$. This poorly defined peak can be understood by taking a closer look at the allowed modes for the waveguide. While the thickness bulk region allows only two modes of propagation, for TE polarization the thickness lies very near the border of allowing a third mode. This results in a weakly damped mode. By shifting the sampling location further from the slit where the modes are excited we see a dramatic drop-off as the mode disappears as seen in Fig. 8.
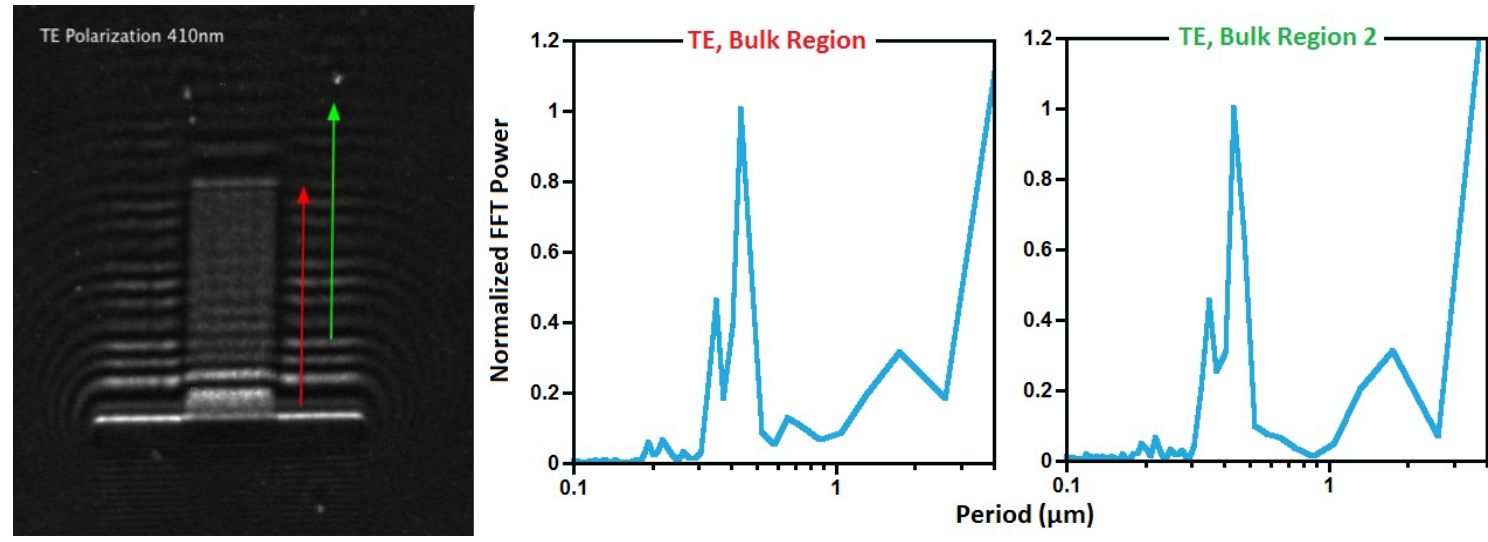

Figure 8. By shifting the data sampling location we observe the disappearance of the strongly suppressed "third" mode. 
The periodograms provide the spacing of the interference pattern, which can be used to determine the propagation constant of the waveguide. To obtain the effective index of the dielectric structure a simple geometrical model is used. The separation of the interference pattern is used to determine the effective index, $\mathrm{N}_{\text {eff, }}$ of the waveguide assuming $\mathrm{x}$ is the distance the guided light travels before it constructively interferes with the incident light.

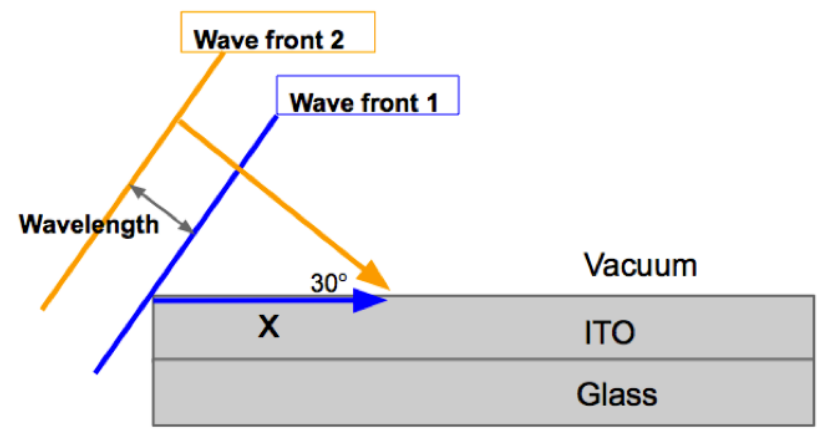

Figure 9: The mode excited by the first wave front is interfered with by the second wave front at some distance $\mathrm{X}$.

$$
\begin{gathered}
\frac{x}{c / N_{\text {eff }}}=\frac{x \cdot \sin 60^{\circ}+\lambda}{c} ; \\
N_{\text {eff }}=\sin 60^{\circ}+\frac{\lambda}{x}
\end{gathered}
$$

where $\lambda$ is the free space wavelength and $60^{\circ}$ is the angle of incidence We can thus determine the effective index of the waveguide for each mode from the experimentally observed interference pattern spacing determined from the periodograms. The effective index is then related to the propagation constant as,

$$
N_{e f f}=\beta \frac{\lambda}{2 \pi}
$$




\section{Simulation}

To simulate the PEEM results we use the radio frequency (RF) module of

COMSOL Multiphysics. COMSOL uses finite element methods to solve Maxwell's equations iteratively for the electromagnetic field on a mesh overlaying the geometry. For our purpose we produce an image of the time averaged real portion of the electric field as we know the photon yield to be proportional to the field as in Eq. (37). Because our structure has symmetry in the y direction we can reduce the model to a 2 -dimensional simulation in the $y=0$ plane. A plane wave is produced incident at an angle of $60^{\circ}$ onto a thin ITO layer with a slit for coupling on a glass substrate as seen in Fig. 10.

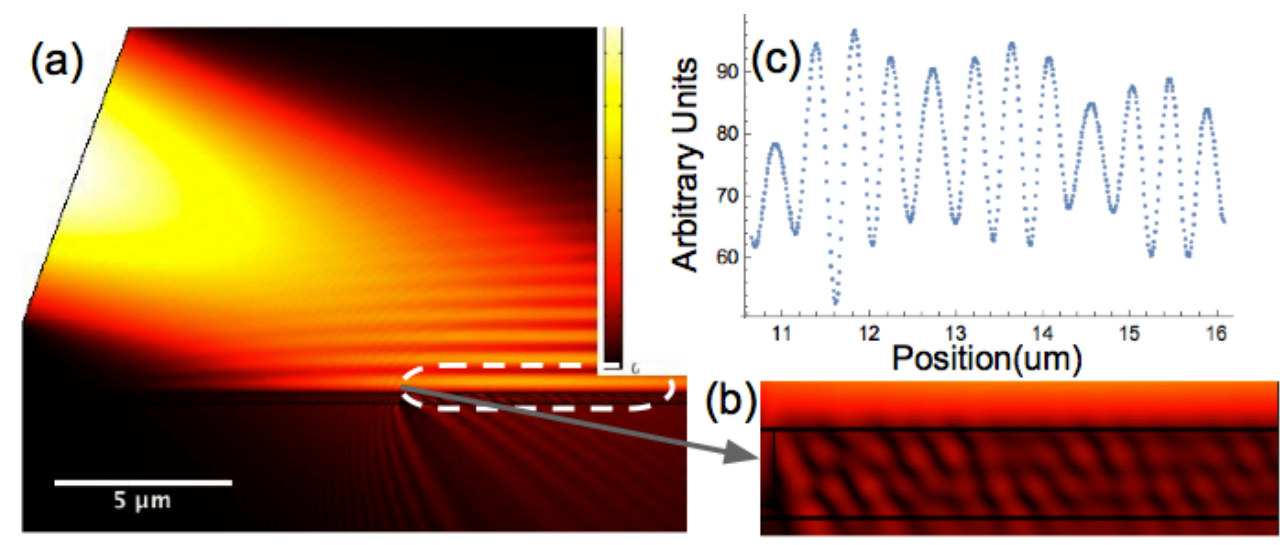

Figure 10. (a) Time averaged electric field in finite element technique simulation using COMSOL multiphysics. (b) Close up of the guiding region supporting two modes. (c) Line graph of the electromagnetic field from the surface of the ITO layer.

The electrons received in PEEM are from the surface of the sample. Therefore we extract a line graph from the vacuum-ITO interface of the time averaged electric field intensity from the simulation. By taking the Fourier transform over the same distance as for the experimental data we find good agreement between the simulation and experimental data for both polarizations as in Fig. 11. Furthermore the relative mode intensities (relative peak heights) of the two main modes of propagation for $\lambda=410 \mathrm{~nm}$ correspond well to those of the experimental data. Note that the mode strongly suppressed mode as discussed in Fig. 8. Is much more apparent 
in simulation. This may indicate the theoretical waveguide thickness used in simulation may be slightly larger than the actual thickness of the ITO layer. The discrepancy at long periods is due to the artifact produced by finite window size in the Fourier Transform. The position of this artifact differs between the experimental and simulated periodograms due to differences in windowing and data resolution. The relevant experimentally observed modes lie outside the range of the window-induced artifacts and can reliably be reproduced in the simulation.

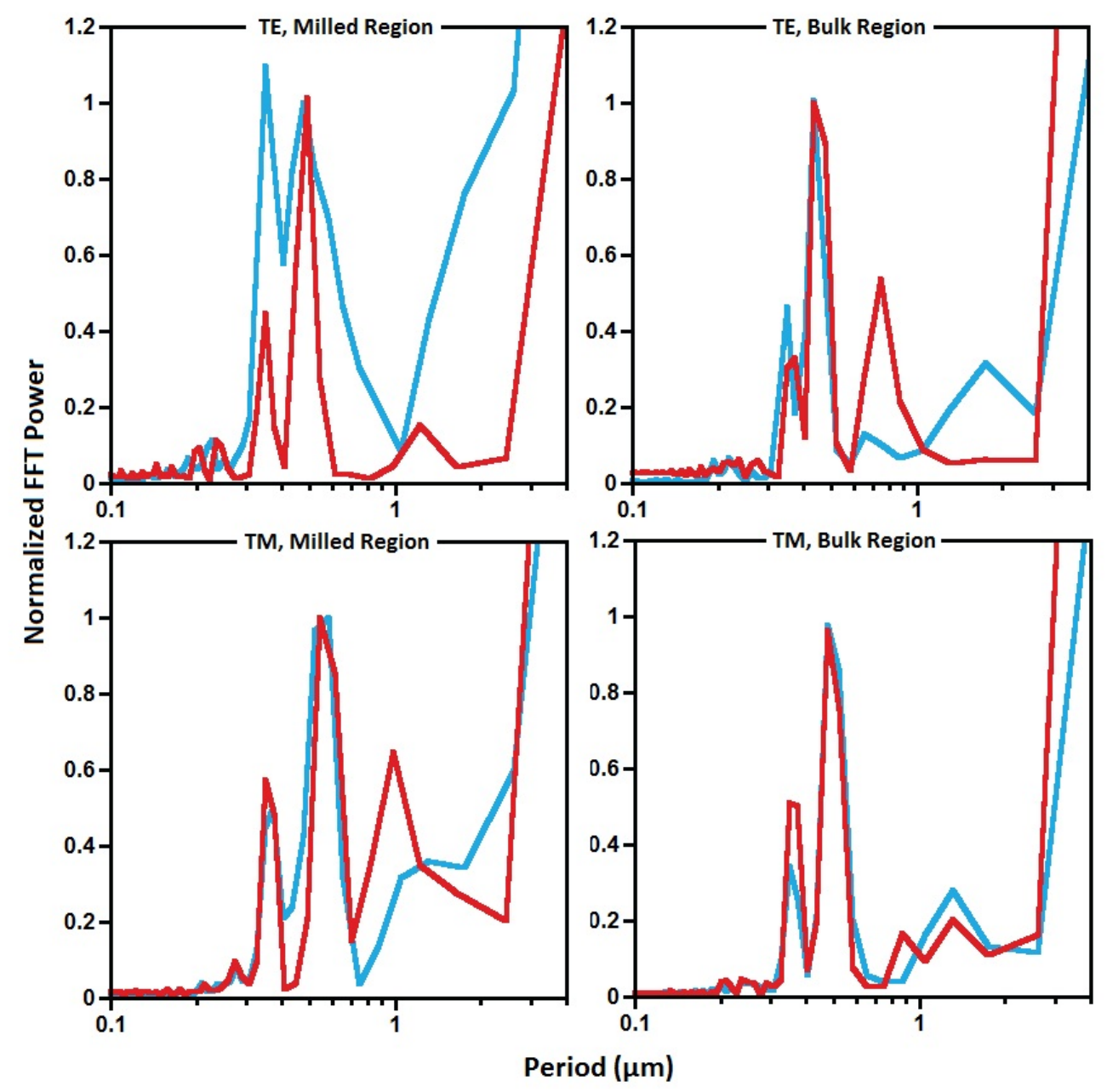

Figure 11: Simulated (red) vs. experimental (blue) data for TE and TM polarizations in the bulk and milled regions at $410 \mathrm{~nm}$ excitation. 
Through simulation we can check the validity of our ray model for the GoosHänchen shift as well. The depth to which the ray penetrates into the cladding is directly tied to how quickly the electromagnetic field decays in the substrate. The decay depth is defined as the distance it takes for the field to decay to $1 / \mathrm{e}$ of its initial value [11]. By exciting a mode directly in the waveguide and taking a cross section we can see the electromagnetic profile across the ITO layer and into the claddings. From this profile the distance it take the field to decay by $1 / \mathrm{e}$ divided by the scale factor $q$ from Eqs. (35)-(36) gives the penetration. The penetration depth then produces a shift through simple geometry of ray tracing as in Eq. (32) [41,42].

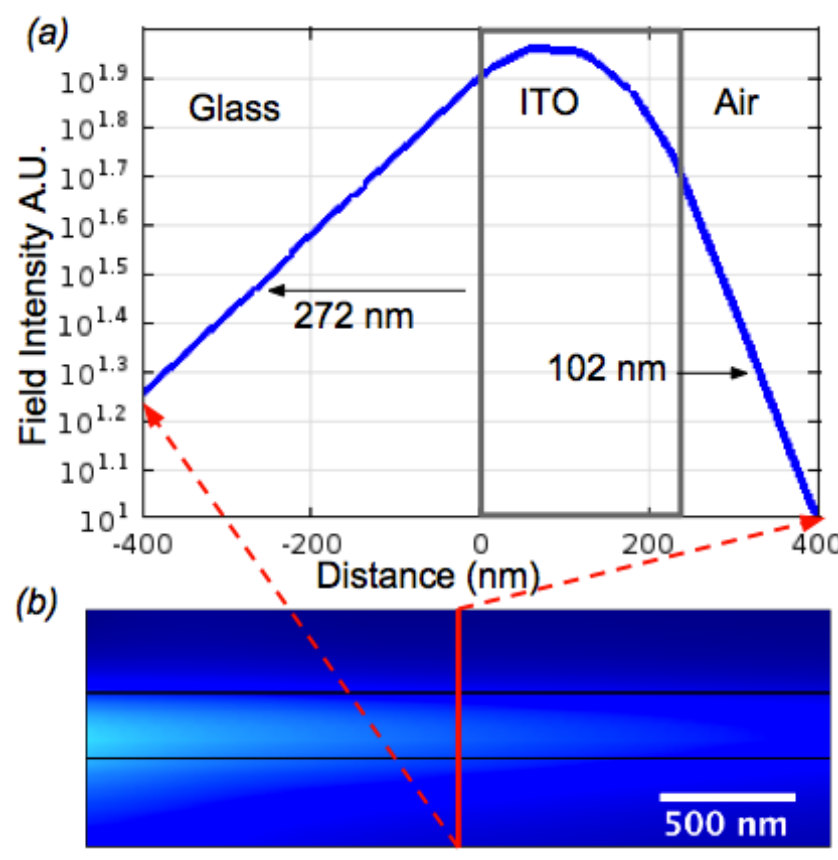

Figure 12. (a) Semi-log line graph of electromagnetic field intensity taken across (b) a COMSOL simulated waveguide with $\mathrm{h}=240 \mathrm{~nm}, \lambda=780 \mathrm{~nm}, \mathrm{n}_{1}=1.78, \mathrm{n}_{2}=1.53, \mathrm{n}_{3}=1$. The graph is used to determine the penetration depth at each interface. 


\section{Goos-Hänchen Shift Results}

We now have several methods of determining the Goos-Hänchen shifts occurring in our waveguide. First, from the measured thickness of our waveguide and refractive indices we may use electromagnetic theory and Artmann's formula as expressed in Eqs. (30)-(31) to calculate a theoretical shift [43]. Second we can experimentally determine the angle of propagation from Fourier analysis of PEEM data and Artmann's formulation. Third we take decay of the electromagnetic field as measure in finite element simulation and use the extended ray model to obtain the shift. A complete summary of the Goos-Hänchen shift values obtained through each method can be seen in tables 1-3.

\begin{tabular}{|l|l|l|l|l|l|l|l|l|}
\hline & \multicolumn{3}{|l|}{ TE $\lambda=410 \mathrm{~nm}$, Mode 1 } & \multicolumn{2}{l|}{ TE $\lambda=410 \mathrm{~nm}$, Mode 2 } \\
\cline { 2 - 9 } & \multicolumn{2}{|l|}{ Milled Region } & \multicolumn{2}{l|}{ Bulk Region } & \multicolumn{2}{l|}{ Milled Region } & \multicolumn{2}{l|}{ Bulk Region } \\
& $\begin{array}{l}\text { Vacuum } \\
\text { Interface }\end{array}$ & $\begin{array}{l}\text { Glass } \\
\text { Interface }\end{array}$ & $\begin{array}{l}\text { Vacuum } \\
\text { Interface }\end{array}$ & $\begin{array}{l}\text { Glass } \\
\text { Interface }\end{array}$ & $\begin{array}{l}\text { Vacuum } \\
\text { Interface }\end{array}$ & $\begin{array}{l}\text { Glass } \\
\text { interface }\end{array}$ & $\begin{array}{l}\text { Vacuum } \\
\text { Interface }\end{array}$ & $\begin{array}{l}\text { Glass } \\
\text { interface }\end{array}$ \\
\hline Artmann & $233 \mathrm{~nm}$ & $307 \mathrm{~nm}$ & $268 \mathrm{~nm}$ & $350 \mathrm{~nm}$ & $128 \mathrm{~nm}$ & $220 \mathrm{~nm}$ & $142 \mathrm{~nm}$ & $215 \mathrm{~nm}$ \\
\hline Simulation & $239 \mathrm{~nm}$ & $313 \mathrm{~nm}$ & $274 \mathrm{~nm}$ & $359 \mathrm{~nm}$ & $131 \mathrm{~nm}$ & $217 \mathrm{~nm}$ & $142 \mathrm{~nm}$ & $210 \mathrm{~nm}$ \\
\hline Experiment & $236 \mathrm{~nm}$ & $311 \mathrm{~nm}$ & $244 \mathrm{~nm}$ & $320 \mathrm{~nm}$ & $127 \mathrm{~nm}$ & $222 \mathrm{~nm}$ & $137 \mathrm{~nm}$ & $214 \mathrm{~nm}$ \\
\hline
\end{tabular}

Table 1: Measurements for TE $\lambda=410 \mathrm{~nm}$ modes in milled and bulk regions.

\begin{tabular}{|l|l|l|l|l|l|l|l|l|}
\hline \multirow{2}{*}{} & \multicolumn{3}{|l|}{ TM $\lambda=410 \mathrm{~nm}$, Mode 1 } & \multicolumn{2}{l|}{ TM $\lambda=410 \mathrm{~nm}$, Mode 2 } \\
\cline { 2 - 9 } & \multicolumn{2}{|l|}{ Milled Region } & \multicolumn{2}{l|}{ Bulk Region } & \multicolumn{2}{l|}{ Milled Region } & \multicolumn{2}{l|}{ Bulk Region } \\
\cline { 2 - 9 } & $\begin{array}{l}\text { Vacuum } \\
\text { Interface }\end{array}$ & $\begin{array}{l}\text { Glass } \\
\text { Interface }\end{array}$ & $\begin{array}{l}\text { Vacuum } \\
\text { Interface }\end{array}$ & $\begin{array}{l}\text { Glass } \\
\text { Interface }\end{array}$ & $\begin{array}{l}\text { Vacuum } \\
\text { Interface }\end{array}$ & $\begin{array}{l}\text { Glass } \\
\text { interface }\end{array}$ & $\begin{array}{l}\text { Vacuum } \\
\text { Interface }\end{array}$ & $\begin{array}{l}\text { Glass } \\
\text { interface }\end{array}$ \\
\hline Artmann & $51 \mathrm{~nm}$ & $169 \mathrm{~nm}$ & $58 \mathrm{~nm}$ & $183 \mathrm{~nm}$ & $54 \mathrm{~nm}$ & $400 \mathrm{~nm}$ & $47 \mathrm{~nm}$ & $220 \mathrm{~nm}$ \\
\hline Simulation & $50 \mathrm{~nm}$ & $172 \mathrm{~nm}$ & $55 \mathrm{~nm}$ & $180 \mathrm{~nm}$ & $55 \mathrm{~nm}$ & $404 \mathrm{~nm}$ & $50 \mathrm{~nm}$ & $226 \mathrm{~nm}$ \\
\hline Experiment & $49 \mathrm{~nm}$ & $164 \mathrm{~nm}$ & $57 \mathrm{~nm}$ & $183 \mathrm{~nm}$ & $56 \mathrm{~nm}$ & $460 \mathrm{~nm}$ & $48 \mathrm{~nm}$ & $237 \mathrm{~nm}$ \\
\hline
\end{tabular}

Table 2: Measurements for TM $\lambda=410 \mathrm{~nm}$ modes in milled and bulk regions. 


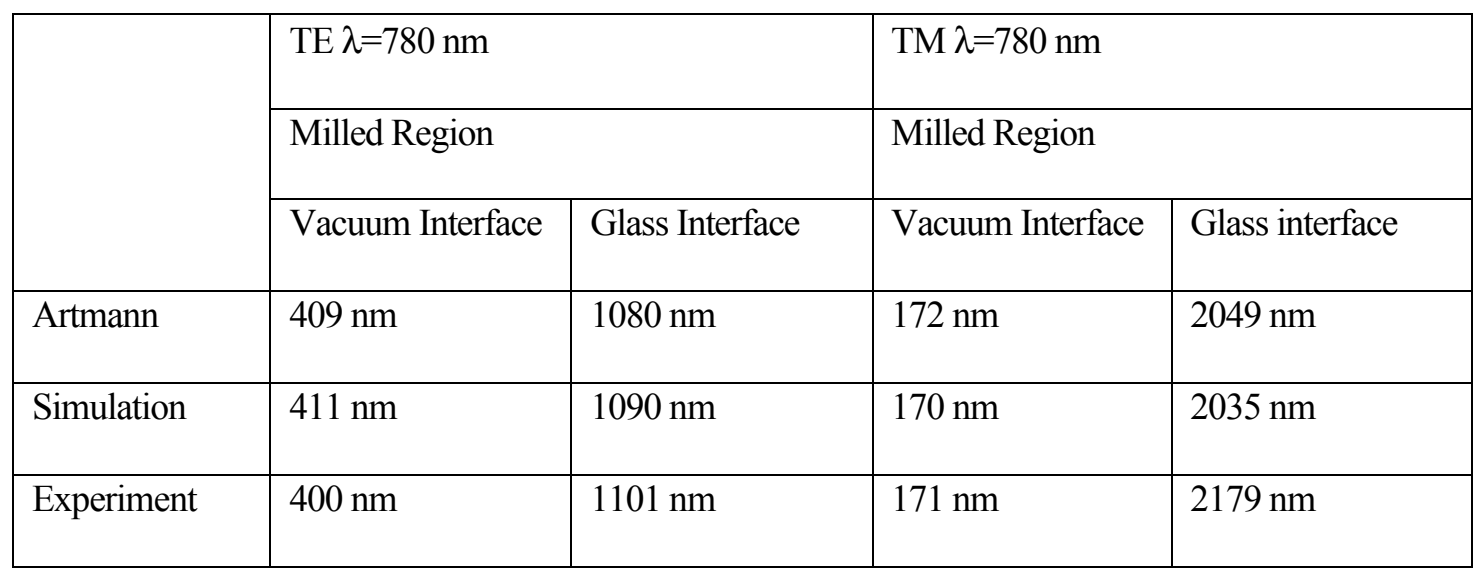

Table 3: Measurements for $\lambda=780 \mathrm{~nm}$ for TE and TM modes in milled regions.

The simulated results provide very good results within $10 \mathrm{~nm}$ of the expected theoretical result. The slight variation can be attributed to the finite meshing and the imperfect absorption at the simulation boundaries. The accuracy to which the Goos-Hänchen shift can be calculated from the experimental data greatly depends on the sensitivity of the shift to changes in the propagation angle. An excellent example can be seen at $780 \mathrm{~nm}$ where the poor resolution due to the reduced signal in 3P-PEEM produces a large discrepancy at the glass interface. However at the vacuum interface the shift the dependence of the shift on the incident angle is very small and thus the values are close to the theoretical results.

A graphical comparison of results for the milled region is seen in Fig. 13. The simulated results are plotted using the theoretical angle and the shift as calculated from the extended ray model. Thus the uncertainty lies in the vertical displacement from the theoretical value. The experimental values are plotted at the theoretical shift and an angel of propagation as determined from the periodograms thus the uncertainty lies in the horizontal direction from the theoretical value. The theoretical shift is calculated from an experimentally determined thickness of the ITO layer of $240 \mathrm{~nm}$ in the milled region. A value of $230 \mathrm{~nm}$ would improve the fit of the experimental data indicating a slight error of $\sim 5 \%$ in the experimental thickness. 

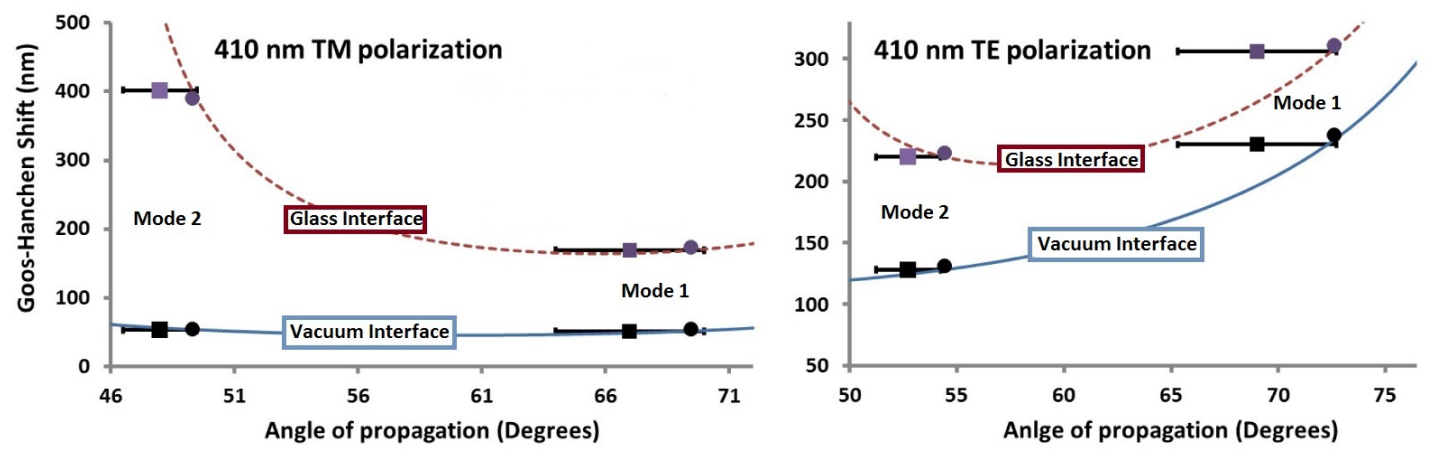

Figure 13. Goos-Hänchen shift vs. angle of propagation at $410 \mathrm{~nm}$ for both interfaces and polarizations in the milled region. Solid line (blue) is the theoretical shift at the air interface. Dashed line (red) is the theoretical shift at the glass interface. Squares represent experimentally determined data points at air interface (black) and glass interface (purple). Similarly circles are data points from simulation.

In Fig. 13 we can see another example of the dependence of the shift on the angle.

For TM polarization Mode 1 has an experimental propagation angle of $\sim 67^{\circ}$ where there is very little change in the shift. Though the uncertainty in the experimental propagation angle is larger for Mode 1 , it obtains a more accurate shift as Mode 2 has a propagation angle of $\sim 48^{\circ}$ where the shift changes very rapidly. The sensitivity to parameters such as incident angle and material index is important in design of optical sensors that incorporate the Goos-Hänchen shift [6]. 


\section{Conclusion}

With the high-resolution capabilities available in PEEM we have successfully measured optical properties of small light confining structures with dimensions on the scale of a wavelength. An extended ray model can accurately represent the propagation of light in our waveguide and this ray model may even be extended to other sub-wavelength structure such as dielectric resonators [44]. Beyond basic science aspects there is currently increased interest in the measurement of Goos-Hänchen shifts. The magnitude of the shift can be greatly increased or even made negative in materials with high imaginary index and in meta-materials [8]. A negative shift is the result of reverse power flow in the cladding and this phenomenon can be exploited to trap or slow down light in the optical wavelength regime $[45,46]$. Slow light devices are critical to the development of optical computing and data storage and have a wide range of potential applications.

While imaginary indices can be difficult to work with in PEEM, however similar results can be obtained in meta-materials such as photonic crystals consisting of periodic modulation of refractive index. These structures can also induce large negative Goos-Hänchen shifts [9]. In future work we hope to show that an ITO based photonic crystal waveguide may be used to slow the propagation of light by taking advantage of the reverse power flow created in the photonic crystal region. 


\section{References}

1. P.K. Tien "Integrated optics and new wave phenomena in optical waveguides," Rev. Mod. Phys. 49(2), 361-420 (1997).

2. F. Goos and H. Hänchen, "Ein neuer und fundamentaler Versuch zur Totalreflexion," Ann. Phys. 6(7-8), 333-346 (1947).

3. C. K. Carniglia and K. R. Brownstein, "Focal shift and ray model for total internal reflection," J. Opt. Soc. Am. 67, 121-122 (1977).

4. J. Li, W, Jing-fang, Z. Le, “Giant Tunable Goos-Hänchen Shifts Based on Prism/Graphene Structure in Terahertz Wave Region, " Photonics Journal, IEEE 6.6 (2014).

5. V. Yallapragada, A. Ravishankar, G. Mulay, G. Agarwal, V. Achanta. “Observation of giant Goos-Hänchen and angular shifts at designed metasurfaces,” Sci. Rep. 6, 19319 (2016).

6. C. Chen, W. Lin, L. Liao, Z. Lin, H Chiang, P. Leung, E. Sijercic, W. Tse, “Optical temperature sensing based on the Goos-Hanchen effect," Appl. Optics 46, 5437, (2007).

7. Y. Wan, Z. Zheng, W. Kong, X. Zhao, J. Liu, "Fiber-to-fiber optical switching based on gigantic Bloch-surface-wave-induced Goos-Hänchen shifts” J. Photon. 5, 7200107 (2013).

8. P. T. Leung, C. W. Chen, and H. P. Chiang, "Large negative Goos-Hanchen shift at metal surfaces," Opt. Commun. 276(2), 206-208 (2007).

9. Jinlong He, Jin Yi, and Sailing He, "Giant negative Goos-Hänchen shifts for a photonic crystal with a negative effective index," Opt. Express 14, 3024-3029 (2006).

10. R. Könenkamp, R.C. Word, G. F. Rempfer, T. Dixon, L. Almaraz and T. Jones, “5.4nm spatial resolution in biological photoemission electron microscopy," Ultramicroscopy 110(7), 899-902 (2010). 
11. H. Kogelnik, Theory of Optical Waveguides in Guided-Wave Optoelectronics, T. Tamir, ed. (Springer-Verlag, 1988).

12. J. J. Burke, Opt. Sci. Newsletter (U. Ariz.) 5, 66 (1971).

13. C. R. Pollock, M. Lipson, Integrated Photonics (Springer-Verlag 2003). Appendix A pp. $(363-365)$

14. X. Liu, Z. Cao, P. Zhu, and Q. Shen, "Solution to causality paradox upon total reflection in optical planar waveguide,” Phys. Rev. E 73, 016615 (2006).

15. A. Gedeon, " Effective thickness of optical waveguides in tunable couplers," J. Opt. Soc. Am. 64 (5), 615-618 (1974).

16. V. Ramaswamy, "Ray model of energy and power flow in anisotropic film waveguides," J. Opt. Soc. Am. 64(10), 1313-1320 (1974).

17. I. Newton, Opticks (Dover, 1952).

18. K. Artmann, "Berechnung der Seitenversetzung des totalreflektierten Strahles," Ann. Physik 6(2), 87-102 (1948).

19. R. H. Renard, "Total reflection: a new evaluation of the Goos-Hänchen Shift," J. Opt. Soc. Am. 54(10), 1190-1197 (1964).

20. B. R. Horowitz and T. Tamir, "Lateral displacement of a light beam at a dielectric interface,”J. Opt. Soc. Am. 61(5), 586-592 (1971).

21. K. W. Chiu, J. J. Quinn, “On the Goos-Hanchen Effect: a simple example of time delay scattering process,” Am. J. Phys. 40, 1847-1851 (1972).

22. E. Brüche, "Elektronenmikroskopische Abbildung mit lichtelektrischen Elektronen," Z. Phys., 86, 448-450 (1933). 
23. E. Bauer, “A brief history of PEEM,” J. Electron Spectrosc. Relat. Phenom. 185, 314, (2012).

24. G. Rempfer, W. Skoczylas, H. Griffith, "Design and performance of a high-resolution photoelectron microscope,” Ultramicroscopy 36(1-3), 196-221 (1991).

25. H. Griffiths, "Photoelectron microscopy - Applications to biological surfaces," Appl. Surf. Sci. 26, 265-279 (1986).

26. D. Habliston, B. Birrell, H. Griffith, G. Rempfer, "Photoelectron imaging of DNA: a study of substrates and contrast,” J. Phys. Chem. 97(12), 3022-3027 (1993).

27. H. Griffith, D Habliston, B. Birrell, P. Skoczylas, K. Hedberg, "Biological photocathodes," Proc. Natl. Acad. Sci. USA, 86(6), 1826-1830, (1989).

28. R. Könenkamp, S. Rouvimov, "Introduction to the special issue: In memoriam Professor Gertrude Fleming Rempfer on occasion of her $100^{\text {th }}$ birthday," Ultramicroscopy 119, 1-4, (2012).

29. L. Zhang, A. Kubo, L. Wang, H. Petek, and T. Seideman, "Imaging of surface plasmon polariton fields excited at a nanometer-scale slit,” Phys. Rev. B 84, 245442 (2011).

30. C. Lemke, T. Leifsner, S. Jaurnik, A. Klick, J. Fiutowski, J. Kjelstrup-Hansen, H. Rubahn, and M. Bauer, "Mapping surface plasmon polariton propagation via counter-propagating light pulses,” Opt. Express 20, 12877 (2012).

31. J. P. S. Fitzgerald, R. C. Word, and R. Könenkamp, "Subwavelength visualization of light in thin film waveguides with photoelectrons," Phys. Rev. B 89, 195129 (2014).

32. J. P. S. Fitzgerald, R. C. Word, S. D. Saliba, and R. Könenkamp, "Photonic near-field imaging in multiphoton photoemission electron microscopy,” Phys. Rev. B 87, 205419 (2013). 
33. J. Feng, A. Scholl, "Photoemission Electron Microscopy," Science of Microscopy. Ed. P. Hawkes, J. Spence, (New York: Springer 1997) 657-695.

34. G. Rempfer, D. Desloge, P. Skoczylas, O. Griffith, "Simultaneous correction of spherical and chromatic aberrations with an electron mirror: An electron optical achromat," Microscopy and Microanalysis. 3, 14-27, (1997).

35. Z. Shao, X. Wu, "Properties of a four-electrode adjustable electron mirror as an aberration corrector,” Rev. Sci. Instrum. 61, 1230 (1990).s

36. J. P. S. Fitzgerald, “Aberation Corrected Electron Microscopy with Photonics Applications" (2015). Dissertation and Thesis. Paper 2192.

37. J. Feng, A. Scholl, “Photoemission Electron Microscopy,” Science of Microscopy. Ed. P. Hawkes, J. Spence, (New York: Springer 1997) 657-695.

38. T. A. F. König, P. A. Ledin, J. Kerszulis, M. A. Mahmoud, M. A. El-Sayed, J. R. Reynolds, and V. V. Tsukruk, "Electrically tunable plasmonic behavior of nanocube-polymer nanomaterials induced by a redox-active electrochromic polymer," ACS Nano. 8 (6), 61826192 (2014).

39. Kim, H. and Horwitz, G. Kushto, A. Pique, Z. H. Kafafi, C. M. Gilmore, and D. B. Chrisey, "Effect of film thickness on the properties of indium tin oxide thin films," J. Appl. Phys. 88, 6021-6025 (2000).

40. R. Schlaf, H. Murata, and Z. H. Kafafi, "Work function measurements on indium tin oxide films,” J. Electron Spectrosc. Relat. Phenom. 120 (1-3), 149-154 (2001).

41. H. Kogelnik and H. P. Weber, "Rays, stored energy and power flow in dielectric waveguides," J. Opt. Soc. Am. 64(2), 174-185 (1974). 
42. P. K. Tien,’Light Waves in Thin Films and Integrated Optics,” Appl. Opt. 10(11), 23952413 (1971).

43. A. Yariv, Optical Electronics (Saunders College Publishing, 1991).

44. D. H. Foster, A. K. Cook, and J. U. Nöckel, “Goos-Hänchen induced vector eigenmodes in a dome cavity," Opt. Lett. 32(12), 1764-1766 (2007).

45. R. Yang, W. Zhu, and J. Li, "Realization of trapped rainbow in 1D slab waveguide with surface dispersion engineering," Opt. Express 23(5), 6326-6335 (2015).

46. K. L. Tsakmakidis, A. D. Boardman, and O. Hess, ““'Trapped rainbow” storage of light in metamaterials," Nature 450, 397-401 (2007). 